\title{
ON MAJORANTS OF SUBHARMONIC AND ANALYTIC FUNCTIONS
}

\author{
FRANTIŠEK WOLF
}

This paper represents a different approach to a whole group of problems connected with majorants of subharmonic functions. The same method has been used previously in order to prove a generalization of the Phragmén-Lindelö theorem. ${ }^{1}$ It seems that the best approach is to prove first Lemma 4 , and then the most important results are easily deducible. Corollary 6 is a generalization of a result of $\mathrm{N}$. Levinson. ${ }^{2}$ His theorem has made me realize the importance of these results.

Lemma 1. If (i) $0<f(x) \leqq 1$ and (ii) $\int_{a}^{b} \log f(x) \cdot d x$ is finite, then

$$
\int_{a}^{b} \log \left|\int_{\xi}^{x} f(y) d y\right| d x
$$

is a continuous function of $\xi$ in $(a, b)$.

We first suppose that $f(x)$ is non-decreasing and that $(0,1)=(a, b)$. We get

$$
\int_{0}^{x} f(y) d y>\int_{x / 2}^{x} f(y) d y \geqq(x / 2) f(x / 2) .
$$

Hence

$$
\begin{aligned}
\int_{0}^{1} \log \left(\int_{0}^{x} f(y) d y\right) d x & >\int_{0}^{1} \log (x / 2) d x+\int_{0}^{1} \log f(x / 2) d x \\
& >2 \int_{0}^{1} \log x \cdot d x+2 \int_{0}^{1} \log f(x) d x \\
& =-2+2 \int_{0}^{1} \log f(x) \cdot d x .
\end{aligned}
$$

If $f(x)$ is replaced by $f(a+(b-a) x)$, we obtain

Presented to the Society, November 22, 1941 under the title On majorants of analytic functions; received by the editors March 2, 1942.

${ }_{1} \mathrm{Cf}$. the end of this paper and Journal of the London Mathematical Society, vol. 14 (1939), p. 208.

${ }^{2}$ Gap and Density Theorems, American Mathematical Society Colloquium Publications, vol. 26, 1940, p. 127, Theorem 43. 


$$
\begin{aligned}
\int_{a}^{b} \log \left(\int_{a}^{x} f(y) d y\right) d x \geqq- & 2(b-a)+(b-a) \log (b-a) \\
& +2 \int_{a}^{b} \log f(x) \cdot d x .
\end{aligned}
$$

Next, if $f(x)$ is general, we form the rearranged non-decreasing function $\bar{f}(x)$ for which

$$
\operatorname{meas} \underset{x}{E}[f(x) \leqq y]=\operatorname{meas} \underset{x}{E}[\bar{f}(x) \leqq y]
$$

for all $y$. We know that ${ }^{3}$

$$
\int_{0}^{1} \log f(x) \cdot d x=\int_{0}^{1} \log \bar{f}(x) \cdot d x,
$$

and that

$$
\left|\int_{x_{0}}^{x} f(y) \cdot d y\right| \geqq \int_{0}^{\left|x-x_{0}\right|} \bar{f}(y) \cdot d y .
$$

Hence, if $x_{0} \subset(0,1)$,

$$
\begin{aligned}
\int_{0}^{1} \log \left|\int_{x_{0}}^{x} f(y) \cdot d y\right| \cdot d x \\
\quad \geqq \int_{0}^{1} \log \left(\int_{0}^{\left|x-x_{0}\right|} \bar{f}(y) \cdot d y\right) \cdot d x \\
\quad=\int_{0}^{x_{0}} \log \left(\int_{0}^{x_{0}-x} \bar{f}(y) \cdot d y\right) \cdot d x+\int_{x_{0}}^{1} \log \left(\int_{0}^{x-x_{0}} \bar{f}(y) \cdot d y\right) d x \\
\quad=\int_{0}^{x_{0}} \log \left(\int_{0}^{x} \bar{f}(y) \cdot d y\right) \cdot d x+\int_{0}^{1-x_{0}} \log \left(\int_{0}^{x} \bar{f}(y) d y\right) d x \\
\geqq 2 \int_{0}^{1} \log \left(\int_{0}^{x} \bar{f}(y) \cdot d y\right) \cdot d x .
\end{aligned}
$$

This, with (2) gives

$$
\begin{aligned}
\int_{0}^{1} \log \left(\int_{x_{0}}^{x} f(y) \cdot d y\right) \cdot d x & \geqq-4+4 \int_{0}^{1} \log \bar{f}(x) \cdot d x \\
& =-4+4 \int_{0}^{1} \log f(x) \cdot d x
\end{aligned}
$$

${ }^{3}$ Hardy, Littlewood, Pólya, Inequalities, Cambridge, 1934, p. 276. 
In a similar fashion we get, for $x_{0} \subset(a, b)$,

$$
\begin{aligned}
\int_{a}^{b} \log \left|\int_{x_{0}}^{x} f(y) d y\right| d x \geqq & -4(b-a)+2(b-a) \log (b-a) \\
& +4 \int_{a}^{b} \log f(x) \cdot d x
\end{aligned}
$$

If $\epsilon>0$ is given, we can find a $\delta>0$ such that

$$
8 \delta-4 \delta \log \delta-4 \int_{x_{0}-\delta}^{x_{0}+\delta} \log f(x) \cdot d x<\epsilon / 3 .
$$

Let us denote by $J_{1}$ the common part of $(a, b)$ and $\left(x_{0}-\delta, x_{0}+\delta\right)$, and by $J_{2}$ the rest of $(a, b)$. Then, by (3), for $\xi \subset J_{1}$

$$
\begin{aligned}
0>\int_{J_{1}} \log \left|\int_{\xi}^{x} f(y) \cdot d y\right| \cdot d x \geqq & -8 \delta+4 \delta \log \delta \\
& +4 \int_{x_{0}-\delta}^{x_{0}+\delta} \log f(x) \cdot d x>-\epsilon / 3 .
\end{aligned}
$$

Further

$$
g_{2}(\xi)=\int_{J_{2}} \log \left|\int_{\xi}^{x} f(y) \cdot d y\right| \cdot d x
$$

is a continuous function for $\xi=x_{0}$ and, hence, there is a $\delta_{1}<\delta$ such that

$$
\left|g_{2}(\xi)-g_{2}\left(x_{0}\right)\right| \leqq \epsilon / 3 \quad \text { for }\left|\xi-x_{0}\right|<\delta_{1} .
$$

Hence, if we call the integral $(1) g(\xi)$, then

$$
\left|g(\xi)-g\left(x_{0}\right)\right|<\epsilon \quad \text { for }\left|\xi-x_{0}\right|<\delta_{1} .
$$

This shows that (1) is a continuous function of $\xi$ at an arbitrary point $x_{0} \subset(a, b)$.

LEMma 2. Given a non-negative $\psi(x) \subset L$, there is a domain $D$, bounded by two continuous curves

$$
C_{1} \equiv y=g_{1}(x), \quad C_{2} \equiv y=g_{2}(x)
$$

and two straight lines $x=x_{0}-a, x=x_{0}+a$, such that, if $x+i y=f\left(r e^{i \theta}\right)$ represents $D$, conformally on the unit circle $r<1$, then

$$
\left|\frac{d x}{d \theta}\right|_{r=1} \geqq \text { const. } \exp [\psi(x)]
$$

on $C_{1}$ and $C_{2}$. 
Further the rectangle

$$
R_{0}:\left[\left|y-y_{0}\right|<a \cdot \log 3 / 2,\left|x-x_{0}\right|<a\right]
$$

is interior to $D$ and

$$
\left|y-y_{0}\right| \leqq c(a), \quad\left|x-x_{0}\right| \leqq a, \quad \text { with } \lim _{a \rightarrow 0} c(a)=0,
$$

contains $D$.

There is no loss of generality in supposing $x_{0}=y_{0}=0$. We define $D$ by constructing its conformal representation on the unit circle. First we define the boundary function of the harmonic function $x(r, \theta)$ so that it satisfies the above condition for the derivative. We define it as the inverse function of

$$
\theta(x)=b \int_{-a}^{x} e^{-\psi(t)} d t
$$

and, for later convenience, we take $b$ such that

$$
b \int_{-a}^{a} e^{-\psi(t)} d t=\theta_{0}<2 \operatorname{arc} \cos 3 / 4<\pi / 3 .
$$

Then the inverse function $x(1, \theta)$, defined in $\left(0, \theta_{0}\right)$ satisfies condition (5) and is continuous and decreasing. Further, we define $x(1, \theta)=a$, for $\theta \subset\left(\theta_{0}, \pi\right) ; x(1, \pi+\theta)=x\left(1, \theta_{0}-\theta\right)$ for $\theta \subset\left(0, \theta_{0}\right)$, and finally $x(1, \theta)=-a$ for $\theta \subset\left(\pi+\theta_{0}, 2 \pi\right)$. In the interval $\left(\pi, \pi+\theta_{0}\right)$ condition (5) is again satisfied. Now, the boundary function is completely defined and

$$
x(r, \theta)=\frac{1-r^{2}}{2 \pi} \int_{0}^{2 \pi} \frac{x(1, \phi) d \phi}{1-2 r \cos (\theta-\phi)+r^{2}} .
$$

The conjugate harmonic function is

$$
y(r, \theta)=-\frac{1}{2 \pi} \int_{0}^{2 \pi} \frac{2 r \sin (\theta-\phi)}{1-2 r \cos (\theta-\phi)+r^{2}} x(1, \phi) d \phi .
$$

By partial integration

$$
y(r, \theta)=\frac{1}{2 \pi} \int_{0}^{2 \pi} \log \left|1-r e^{i(\theta-\phi)}\right| d x(1, \phi) .
$$

Since $x$ is constant in some intervals, we get, using the above definition of $x(1, \phi)$, 


$$
\begin{aligned}
y(r, \theta)=\frac{1}{2 \pi} \int_{0}^{\theta_{0}}\left[\log \left|1-r e^{i(\theta-\phi)}\right|\right. & \\
& \left.-\log \left|1+r e^{i\left(\theta-\theta_{0}+\phi\right)}\right|\right] d x(1, \phi) .
\end{aligned}
$$

Now, the required conformal representation is given by $x(r, \theta)$ $+i y(r, \theta)$. The boundary curve of $D$ in the parametric form is $x(1, \theta)+i y(1, \theta)$, and we shall investigate it.

If $\theta \subset\left(0, \theta_{0}\right)$, then $x(1, \theta)$ runs from $-a$ to $+a$ and, since $\left|1-e^{i(\theta-\phi)}\right|$ $<1$ for $\theta, \phi \in\left(0, \theta_{0}\right) \subset(0, \pi / 3)$ (cf. (8)),

$$
\begin{aligned}
y(1, \theta) & \leqq-\frac{1}{2 \pi} \int_{0}^{\theta_{0}} \log \left|1+e^{i\left(\theta-\theta_{0}+\phi\right)}\right| d x \\
& \leqq-\frac{1}{2 \pi} \log \left|1+e^{i \theta_{0}}\right| \int_{-a}^{a} d x \\
& =-(a / \pi) \log 2 \cos \theta_{0} / 2<-(a / \pi) \log 3 / 2 .
\end{aligned}
$$

The first term $I_{1}$ on the right in (9) is more difficult to dispose of. We have

$$
\begin{aligned}
I_{1}= & \frac{1}{2 \pi} \int_{0}^{\theta_{0}} \log \left|2 \sin \frac{\theta-\phi}{2}\right| \cdot d x(1, \phi) \\
= & \frac{1}{2 \pi} \int_{0}^{\theta_{0}} \log \frac{2 \sin (\theta-\phi) / 2}{\theta-\phi} \cdot d x(1, \phi) \\
& \quad+\frac{1}{2 \pi} \int_{0}^{\theta_{0}} \log |\theta-\phi| \cdot d x(1, \phi)=I_{1}^{\prime}+I_{1}^{\prime},
\end{aligned}
$$

Here, $I_{1}^{\prime}$ is evidently a continuous function of $\theta$ and

$$
0>I_{1}>-\frac{a}{\pi} \log \frac{2 \sin \theta_{0} / 2}{\theta_{0}} .
$$

By means of (7), we change variables in $I_{1}^{\prime \prime}$ and obtain

$$
I_{1}^{\prime \prime}=\frac{1}{2 \pi} \int_{-a}^{a} \log \left|b \int_{\xi}^{x(\theta)} e^{-\psi(\alpha)} \cdot d \alpha\right| \cdot d \xi .
$$

By Lemma 2, this is a continuous function of $x$ and therefore also of $\theta$ and from (3) we get

$$
0>I_{1}^{\prime \prime}>-\frac{2 a}{\pi}+\frac{a \log 2 a}{\pi}+\frac{1}{\pi} \int_{-a}^{a} \log \left|b e^{-\psi(x)}\right| d x .
$$


If we combine the last inequalities and introduce the abbreviation $c(a)$, we get

with

$$
-\frac{a}{\pi} \log 3 / 2>y(1, \theta)>-c(a), \quad \theta \subset\left(0, \theta_{0}\right),
$$

$$
\lim _{a \rightarrow 0} c(a)=0 .
$$

From (9) it is easy to deduce that

$$
y(r, \theta+\pi)=-y\left(r, \theta_{0}-\theta\right)
$$

and we obtain

$$
(a / \pi) \log 3 / 2<y(1, \theta) \leqq c(a), \text { for } \theta \subset\left(\pi, \pi+\theta_{0}\right) .
$$

Using the same notation we shall prove the following lemma.

Lemma 3. If $\psi\left(x_{0}-a\right), \psi\left(x_{0}+a\right)$ are finite, then every subharmonic function $\alpha(x, y)$, defined in $D$, which satisfies

$$
\alpha(x, y) \leqq e^{\psi(x)}, \quad|x|<a,
$$

has an upper bound in $R[|y|<a \cdot \log 3 / 2,|x|<a]$ which depends only on $\psi(x)$.

If we represent $D$ conformally on the unit circle $C$, we get from $\alpha(x, y)$ a subharmonic function $\alpha(r, \theta)$ defined in $C$. There, it must be less than any harmonic function with boundary values not less than those of $\alpha(r, \theta)$. By (10), these are not greater than $\exp \{\psi(x(1, \theta))\}$, where $x(1, \theta)$ has been defined in the preceding lemma. Such a harmonic function is the Poisson integral of $\exp \{\psi(x(1, \theta))\}$. We have to show that this Poisson integral is not identically equal to $\infty$. A sufficient condition is

$$
\int_{0}^{2 \pi} \exp \{\psi(x(1, \theta))\} \cdot d \theta<\infty .
$$

In view of the definition of $x(1, \theta)$, this integral is obviously less than

$$
\left|\int_{-a}^{a} e^{\psi(x)} \frac{d \theta}{d x} d x\right|+\int_{\theta_{0}}^{\pi} e^{\psi(a)} d \theta+\left|\int_{a}^{-a} e^{\psi(x)} \frac{d \theta}{d x} d x\right|+\int_{\pi+\theta_{0}}^{2 \pi} e^{\psi(-a)} d \theta,
$$

and using (5), also less than

$2 b \int_{-a}^{a} e^{\psi(x)} e^{-\psi(x)} d x+\pi\left(e^{\psi(a)}+e^{\psi(-a)}\right) \leqq 4 a b+\pi\left(e^{\psi(a)}+e^{\psi(-a)}\right)<\infty$. 
Hence $\alpha(x, y)$ is, inside of $D$, less than a finite harmonic function depending only on $\psi(x)$. In the domain $D$, completely interior to $R$, it is therefore bounded from above by a constant depending only on $\psi(x)$.

Theorem. If (i) $\alpha(x, y)$ is subharmonic in $R[|x|<c,|y|<d]$ and (ii) $\alpha(x, y)<e^{\psi(x)}, \psi(x) \subset L, x \subset(-c, c), \psi(-c)<\infty, \psi(c)<\infty$, then for any $\delta$, such that $0<\delta<d$, there is an upper bound $C$ for $\alpha(x, y)$ in $D_{0}[|x|<c,|y|<d-\delta]$ dependent only on $\delta$ and $\psi(x)$, but independent of the particular $\alpha(x, y)$.

In view of the Lemma 3 , it is sufficient to show that there is an $a$ and a finite number of domains $D_{k}$ satisfying the conditions of this lemma, and, such that $D_{k}$ contains (cf. (6)) $R_{k}\left[\left|x-x_{k}\right|<a,\left|y-y_{k}\right|\right.$ $\leqq c(a)]$, is contained in $R$, and that $\sum R_{k}$ covers completely $D_{0}$. Since $\alpha(x, y)$ has a finite upper bound in every $R_{k}$, it must be so also in $D_{0}$. And the upper bound will depend only on $\psi(x)$ and $D_{0}$.

We determine $a>0$ such that $c(a)-a \log 3 / 2<\delta / 2$. If we define $D^{*}[|x|<c,|y|<d-\delta / 2]$, then $D_{0} \subset D^{*} \subset R$, and, if $R_{k} \subset D^{*}$, then the corresponding $D_{k} \subset R$ (cf. (3.2)). Since $R_{k}$ can be any rectangle, of the above size, in $D^{*}$ and such that $\psi\left(x_{k} \pm a\right)$ is finite, it is evident that we can find a finite number of them covering completely $D_{0}$. The theorem is proved.

Corollary: Let $f(z)$ be a function, analytic in $R[|x|<c,|y|<d]$, such that

$$
|f(z)| \leqq M(x) .
$$

If $\int_{-c}^{c} \log ^{+} \log ^{+} M(x) d x<\infty$, then for every domain $D_{0}$ completely interior to $R$, there exists a $\phi$ depending only on $D_{0}$ and $M(x)$, such that

$$
|f(z)| \leqq \phi \quad \text { for } z \subset D_{0} .
$$

There is no loss of generality to suppose $M(x)>e$. and then the sign + can be omitted over the log signs. If $f(z)$ is analytic, then $\log |f(z)|$ is subharmonic and the result follows from the preceding theorem.

Nils Sjöberg has proved the following theorem:4

Let $M(\theta)$ be given and $0<\epsilon<1$. In order that the class of subharmonic functions, defined in $|z|<1$, which satisfy in $1-\epsilon \leqq|z|<1$

$$
\left|\mu\left(r e^{i \theta}\right)\right| \leqq M(\theta),
$$

${ }^{4}$ Comptes Rendus du Congrès des Mathématiques à Helsinfors, 1938. 
should be bounded from above in every circle $|z| \leqq r_{0}<1$, it is sufficient that

$$
\int_{-\pi}^{\pi} \log ^{+} M(\theta) \cdot d \theta<\infty .
$$

This theorem can easily be deduced from our result. By $\zeta=\log z$, we straighten out the concentric circles, and $1-\epsilon \leqq|z|<1$ corresponds to $\log (1-\epsilon) \leqq R(\zeta)<0$. In this strip $\mu(\zeta) \leqq M(\theta), \theta=\Im(\zeta)$. Hence, if $M\left(\theta_{0}\right)<\infty$, then, by our result, the class of subharmonic functions $\mu(\zeta)$ will be bounded in $\theta_{0} \leqq \arg z=\theta \leqq \theta_{0}+2 \pi$ and $\log (1-\epsilon / 2)$ $\leqq R(\zeta) \leqq \log (1-\epsilon / 4)$. Hence the same is true of $\mu(z)$ in $1-\epsilon / 2 \leqq|z|$ $\leqq 1-\epsilon / 4$. But the class of subharmonic functions must have the same upper bound in $|z| \leqq 1-\epsilon / 4$. The theorem is proved.

Similarly we can prove a generalization of the Phragmén-Lindelöf theorem $:^{5}$

If: (i) $f(z)$ is analytic in $\Im(z)>0$, (ii) its boundary values on $\Im(z)=0$ are in absolute value less than 1 , (iii) there are two sequences $r_{k} \rightarrow \infty$ and $\epsilon_{k} \rightarrow 0$ such that

$$
|f(z)| \leqq \exp \left\{\epsilon_{k} r_{k} e^{\psi(\theta)}\right\}, \quad \psi(\theta) \subset L,
$$

for $r_{k}(1-\delta)<|z|<r_{k}$, then

$$
|f(z)| \leqq 1 \quad \text { for } \Im(z)>0 .
$$

From condition (iii) it follows that

$$
\log \left|f\left(\boldsymbol{r}_{k} z\right)\right| / \epsilon_{k} r_{k} \leqq e^{\psi(\theta)},
$$

for $1-\delta<|z|<1$. By condition (ii), we can suppose $\psi(0)=\psi(\pi)=0$. In the same way as in the preceding we deduce the existence of a $\phi$, such that

$$
\log \left|f\left(r_{k} z\right)\right| / \epsilon_{k} r_{k}<\phi \quad \text { for } 1-\delta / 2<|z|<1-\delta / 4,
$$

or

$$
|f(z)| \leqq \exp \left\{\epsilon_{k} \phi r_{k}\right\}, \quad(1-\delta / 2) r_{k}<|z|<(1-\delta / 4) r_{k} .
$$

Now we use the Phragmén-Lindelöf theorem in its classical form ${ }^{6}$ to deduce the desired result.

UNIVERSITY OF CALIForNia

${ }^{5}$ Journal of the London Mathematical Society, vol. 14 (1939), p. 208.

${ }^{6}$ R. Nevanlinna, Eindeutige analytische Funktionen, Berlin, 1936, p. 43. 